\title{
Article \\ Thrombopoietin Contributes to Enhanced Platelet Activation in Patients with Type 1 Diabetes Mellitus
}

\author{
Ornella Bosco ${ }^{1,+}{ }^{\dagger}$, Barbara Vizio ${ }^{1,+}$, Gabriella Gruden ${ }^{1}$, Martina Schiavello ${ }^{1} \oplus$, Bartolomeo Lorenzati ${ }^{2}$, \\ Paolo Cavallo-Perin ${ }^{1}$, Isabella Russo ${ }^{3, *}$ (i) , Giuseppe Montrucchio ${ }^{1, *, \neq}$ and Enrico Lupia ${ }^{1, *, \neq(1)}$ \\ 1 Department of Medical Sciences, University of Turin, 10126 Turin, Italy; ornella.bosco@unito.it (O.B.); \\ barbara.vizio@unito.it (B.V.); gabriella.gruden@unito.it (G.G.); martina.schiavello@unito.it (M.S.); \\ paolo.cavalloperin@gmail.com (P.C.-P.) \\ 2 Emergency Medicine, A.O. S. Croce e Carle, 12100 Cuneo, Italy; lorebato@gmail.com \\ 3 Department of Clinical and Biological Sciences, University of Turin, 10043 Orbassano, Italy \\ * Correspondence: isabella.russo@unito.it (I.R.); giuseppe.montrucchio@unito.it (G.M.); \\ enrico.lupia@unito.it (E.L.) \\ + These Authors equally contributed to the work. \\ $\ddagger$ These Authors equally contributed to the work.
}

Citation: Bosco, O.; Vizio, B.; Gruden, G.; Schiavello, M.; Lorenzati, B.; Cavallo-Perin, P.; Russo, I.; Montrucchio, G.; Lupia, E. Thrombopoietin Contributes to Enhanced Platelet Activation in Patients with Type 1 Diabetes Mellitus. Int. J. Mol. Sci. 2021, 22, 7032. https://doi.org/10.3390/ ijms22137032

Academic Editor: Manuel VázquezCarrera

Received: 9 June 2021

Accepted: 25 June 2021

Published: 29 June 2021

Publisher's Note: MDPI stays neutral with regard to jurisdictional claims in published maps and institutional affiliations.

Copyright: (c) 2021 by the authors Licensee MDPI, Basel, Switzerland. This article is an open access article distributed under the terms and conditions of the Creative Commons Attribution (CC BY) license (https:// creativecommons.org/licenses/by/ $4.0 /)$.

\begin{abstract}
Atherosclerotic cardiovascular disease is the major cause of morbidity and mortality in patients with type 1 diabetes mellitus (T1DM). Enhanced platelet reactivity is considered a main determinant of the increased atherothrombotic risk of diabetic patients. Thrombopoietin (THPO), a humoral growth factor able to stimulate megakaryocyte proliferation and differentiation, also modulates the response of mature platelets by enhancing both activation and binding to leukocytes in response to different agonists. Increased THPO levels have been reported in different clinical conditions characterized by a generalized pro-thrombotic state, from acute coronary syndromes to sepsis/septic shock, and associated with elevated indices of platelet activation. To investigate the potential contribution of elevated THPO levels in platelet activation in T1DM patients, we studied 28 T1DM patients and 28 healthy subjects. We measured plasma levels of THPO, as well as platelet-leukocyte binding, P-selectin, and THPO receptor (THPOR) platelet expression. The priming activity of plasma from diabetic patients or healthy subjects on platelet-leukocyte binding and the role of THPO on this effect was also studied in vitro. T1DM patients had higher circulating THPO levels and increased platelet-monocyte and platelet-granulocyte binding, as well as platelet P-selectin expression, compared to healthy subjects, whereas platelet expression of THPOR did not differ between the two groups. THPO concentrations correlated with platelet-leukocyte binding, as well as with fasting glucose and Hb1Ac. In vitro, plasma from diabetic patients, but not from healthy subjects, primed platelet-leukocyte binding and platelet P-selectin expression. Blocking THPO biological activity using a specific inhibitor prevented the priming effect induced by plasma from diabetic patients. In conclusion, augmented THPO may enhance platelet activation in patients with T1DM, potentially participating in increasing atherosclerotic risk.
\end{abstract}

Keywords: type 1 diabetes mellitus; platelet-leukocyte adhesion; platelet activation markers; thrombopoietin; atherosclerotic cardiovascular diseases

\section{Introduction}

Cardiovascular disease (CVD) is the major cause of morbidity and mortality in patients with type 1 diabetes mellitus (T1DM) [1]. The rising global incidence of T1DM with an even earlier age of onset leads to a longer exposure to the disease and a greater risk of premature CVD with microvascular and macrovascular complications that accelerate the process of atherosclerosis [2,3].

Pro-thrombotic factors, together with other pathophysiologic mechanisms, which include hyperglycemia, insulin resistance, hyperlipidemia, and a generalized pro- 
inflammatory state, play a determinant role in sustaining the accelerated atherosclerosis and increased cardiovascular risk profile of diabetic patients [4,5]. Enhanced platelet reactivity, together with endothelial dysfunction, increased levels of coagulation factors, and impaired fibrinolysis, has been associated with the elevated atherothrombotic risk of diabetic patients, and oral antiplatelet therapy has become a key treatment for reducing the long-term risk of ischemic events in DM patients [6-8]. Interestingly, DM patients remain at high risk of major cardiovascular events even on antiplatelet treatment, probably because of a greater prevalence, compared to non-diabetics, of inadequate platelet inhibition or "resistance" to antiplatelet therapy. The use of more potent and prolonged antiplatelet therapies reduces the rates of drug resistance and the recurrence of ischemic events, but the increase in bleeding complications represents a major concern [9].

Thrombopoietin (THPO) is a humoral growth factor constitutively produced by the liver and kidneys and promotes megakaryocyte proliferation and differentiation. The binding with its receptor c-Mpl (THPOR), mainly expressed on platelets and megakaryocytes, clears THPO from circulation and allows it to exert its biological functions [10,11].

Previous studies demonstrated that THPO directly modulates the response of mature platelets to several stimuli and thereby their homeostatic potential [12,13]. In particular, THPO, does not induce platelet aggregation per se, but can enhance both platelet activation and platelet-leukocyte adhesion in response to various agonists [12-14]. Increased THPO levels have been shown in different clinical conditions characterized by a pro-thrombotic state, from acute coronary syndromes to sepsis/septic shock and cigarette smoking, and are associated with elevated indices of platelet activation [15-17].

The aim of this study was to explore the potential contribution of THPO in enhancing platelet reactivity in patients with T1DM.

\section{Results}

\subsection{Study Subjects}

We enrolled 28 type 1 diabetic patients and 28 healthy subjects, who were matched in a 1:1 ratio for age and gender to cases.

Table 1 shows demographic and clinical data of both patients and controls.

Table 1. Subject characteristics.

\begin{tabular}{|c|c|c|}
\hline Characteristics & $\begin{array}{l}\text { Controls } \\
(n=28)\end{array}$ & $\begin{array}{c}\text { T1DM } \\
(n=28)\end{array}$ \\
\hline Age (years), median (IQR) & $26.00(23.00-36.00)$ & $28.00(22.25-36.75)$ \\
\hline Gender $(n)$, female/male & $10 / 18$ & $10 / 18$ \\
\hline WBC $\left(10^{9} / \mathrm{L}\right)$, median (IQR) & $5.38(4.73-5.63)$ & $6.15(4.64-7.27)$ \\
\hline Monocytes $\left(10^{9} / \mathrm{L}\right)$, mean $\pm \mathrm{SE}$ & $0.37 \pm 0.02$ & $0.40 \pm 0.04$ \\
\hline Granulocytes $\left(10^{9} / \mathrm{L}\right)$, median (IQR) & $2.94(2.56-3.39)$ & $3.17(2.07-4.78)$ \\
\hline Platelets $\left(10^{9} / \mathrm{L}\right)$, mean $\pm \mathrm{SE}$ & $231.60 \pm 7.75$ & $235.80 \pm 14.58$ \\
\hline $\mathrm{MPV}(\mathrm{fL})$, mean $\pm \mathrm{SE}$ & $10.42 \pm 0.15$ & $10.74 \pm 0.20$ \\
\hline $\mathrm{PDW}(\mathrm{fL})$, mean $\pm \mathrm{SE}$ & $12.52 \pm 0.32$ & $12.98 \pm 0.37$ \\
\hline Total cholesterol $(\mathrm{mg} / \mathrm{dL})$, mean $\pm \mathrm{SE}$ & $186.00 \pm 5.88$ & $175.40 \pm 5.18$ \\
\hline $\mathrm{HDL}$ cholesterol $(\mathrm{mg} / \mathrm{dL})$, mean $\pm \mathrm{SE}$ & $56.75 \pm 2.72$ & $51.36 \pm 2.30$ \\
\hline LDL cholesterol $(\mathrm{mg} / \mathrm{dL})$, mean $\pm \mathrm{SE}$ & $112.60 \pm 6.10$ & $111.40 \pm 4.40$ \\
\hline Triglycerides (mg/dL), median (IQR) & $67.50(57.25-81.50)$ & $58.00(48.00-78.00)$ \\
\hline $\mathrm{BMI}\left(\mathrm{Kg} / \mathrm{m}^{2}\right)$, mean $\pm \mathrm{SE}$ & $21.90 \pm 0.59$ & $21.57 \pm 0.39$ \\
\hline Diabetes duration (years), mean $\pm \mathrm{SE}$ & n.a. & $13.31 \pm 1.55$ \\
\hline Fasting glucose (mg/dL), median (IQR) & $90.00(85.25-100.80)$ & $164.00(130.00-217.00)^{* * *}$ \\
\hline $\mathrm{HbA1c}(\mathrm{mmol} / \mathrm{mol})$, median (IQR) & $36.00(34.00-38.00)$ & $59.00(51.25-66.75) * * *$ \\
\hline
\end{tabular}

$\mathrm{BMI}=$ body mass index; $\mathrm{HDL}=$ high-density lipoprotein; $\mathrm{IQR}=$ interquartile range; $\mathrm{LDL}=$ low-density lipoprotein; $\mathrm{MPV}=$ mean platelet volume; n.a. = not applicable; $\mathrm{PDW}=$ platelet distribution width; T1DM $=$ type 1 diabetes mellitus; $\mathrm{WBC}=$ white blood cell. ${ }^{* * *} p<0.001 \mathrm{vs}$. controls, obtained by Mann-Whitney test. 
Type 1 diabetic patients and healthy subjects did not differ regarding demographic characteristics. Platelet, leukocyte, monocyte, and granulocyte counts were not significantly different in the two groups, neither were mean platelet volume (MPV) and platelet distribution width (PDW). Finally, total, HDL and LDL cholesterol levels and triglycerides were similar between the two groups, whereas type 1 diabetic patients had, as expected, higher fasting glucose and $\mathrm{HbA1c}$ concentrations than healthy subjects.

\subsection{THPO Concentrations and Platelet THPOR Expression in Type 1 Diabetes Mellitus Patients}

Plasma THPO concentrations were significantly higher in patients with T1DM than in healthy controls (median (range): $24.45 \mathrm{pg} / \mathrm{mL}$ (16.97-57.60) vs. $21.23 \mathrm{pg} / \mathrm{mL}$ (11.70-35.09)) (Figure 1A). In contrast, there were no differences in the surface expression of THPOR on platelets between T1DM patients and controls (median (range): 68.95\% (12.00-92.90\%) vs. $71.20 \%(35.40-89.40 \%)$ ) (Figure 1B).

A

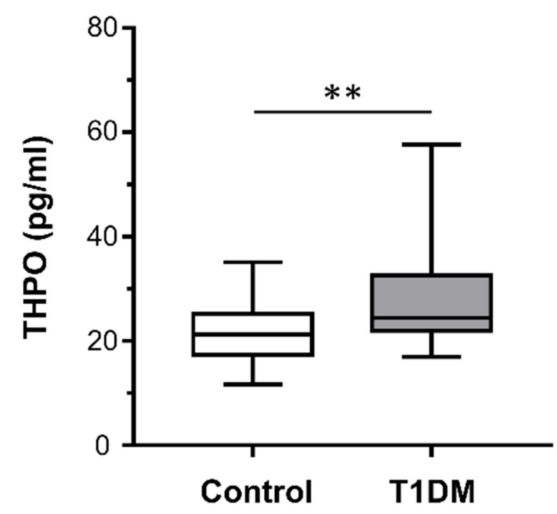

C

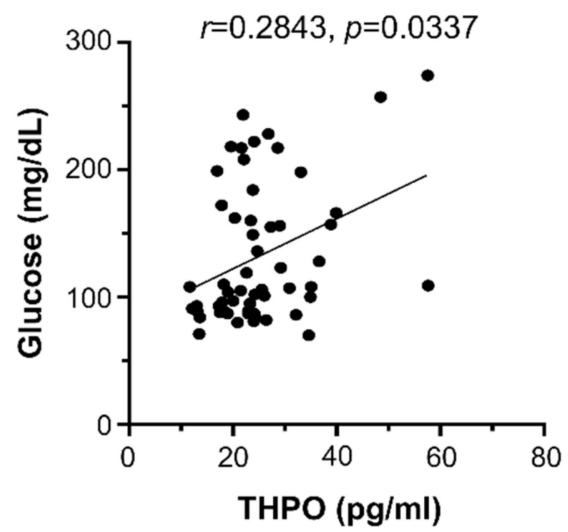

B

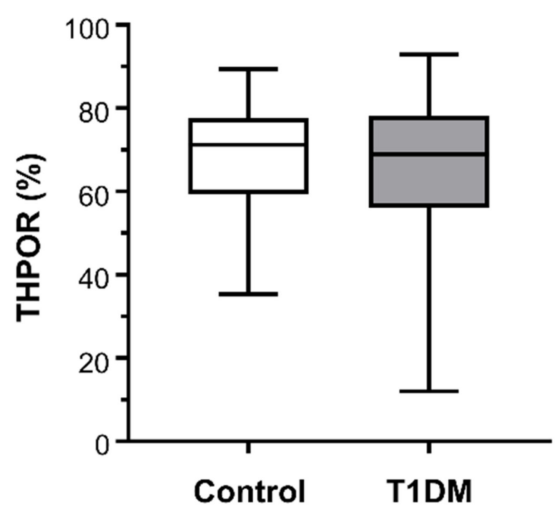

D

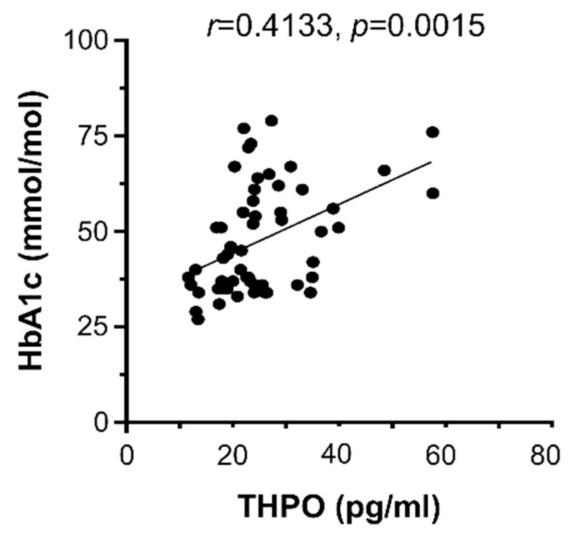

Figure 1. Circulating thrombopoietin (THPO) levels, measured by ELISA (A), and THPO receptor (R) expression, detected ex vivo by flow cytometry $(\mathbf{B})$, in healthy controls $(n=28)$ and type 1 diabetes mellitus (T1DM) patients $(n=28)$. Data were represented as median (range). $p$-values by Mann-Whitney test $\left((\mathbf{A}){ }^{* *} p<0.01\right.$; (B) not statistically significant). Correlation of THPO plasma levels with fasting plasma glucose (C) and HbA1c (D). Analyses by Spearman correlation tests; correlation coefficient $\mathrm{r}$ - and $p$-values are shown.

We found no correlation between THPO concentrations and either platelet count or markers of augmented platelet turnover (MPV and PDW). On the contrary, THPO concentrations positively correlated with both fasting plasma glucose $(r=0.2843, p=0.0337$; Figure 1C) and HbA1c $(r=0.4133, p=0.0015$; Figure 1D). 


\subsection{Ex Vivo Platelet Activation Studies}

Patients with T1DM had significantly higher percentages of platelet-monocyte (median (range): $34.50 \%(13.40-45.20 \%)$ vs. $23.50 \%(13.10-31.00 \%)$; Figure $2 \mathrm{~A})$ and plateletgranulocyte binding (median (range): $10.35 \%$ (5.70-31.30\%) vs. $7.35 \%(2.60-25.20 \%)$; Figure 2B), as well as increased surface expression of P-selectin on platelets (median (range): $2.15 \%(1.00-4.30 \%)$ vs. $1.70 \%(1.10-3.30 \%)$; Figure $2 \mathrm{C})$ compared with healthy subjects.

A

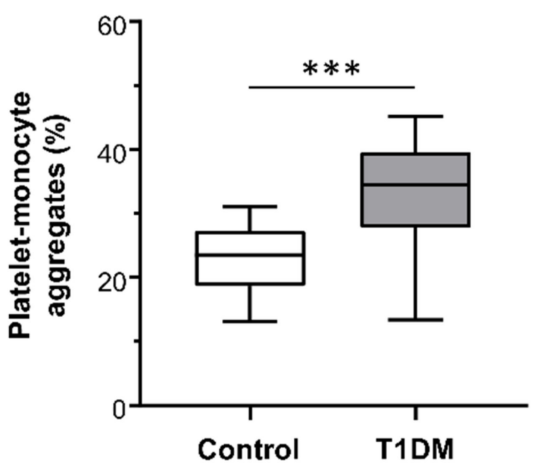

B

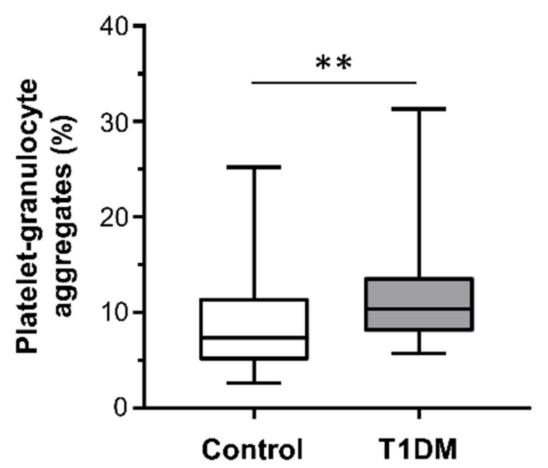

C

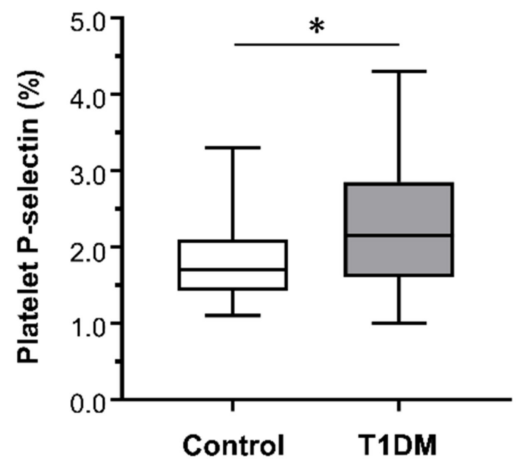

Figure 2. Platelet-monocyte aggregates (A), platelet-granulocyte aggregates (B), and platelet P-selectin expression (C) detected ex vivo, by flow cytometry, in healthy controls $(n=28)$ and type 1 diabetes mellitus (T1DM) patients $(n=28)$. Data were represented as median (range). $p$-values by Mann-Whitney test $\left((\mathbf{A}){ }^{* *} p<0.001,(\mathbf{B}){ }^{* *} p<0.01\right.$, and $\left.(\mathbf{C}) * p<0.05\right)$.

THPO plasma levels significantly correlated with ex vivo platelet-monocyte aggregation $(r=0.3000, p=0.0247$; Figure 3A) and platelet-granulocyte binding $(r=0.2955$, $p=0.0271$; Figure $3 \mathrm{~B})$, but not with platelet P-selectin expression $(r=0.2411, p=0.0735$; not shown).

A

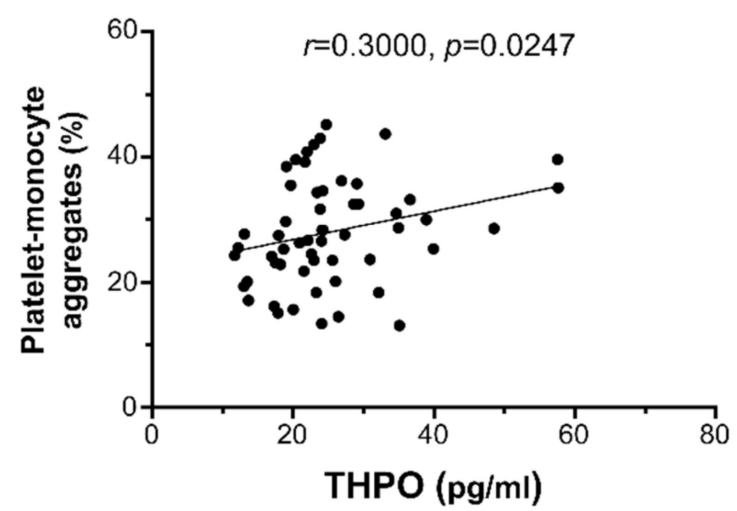

B

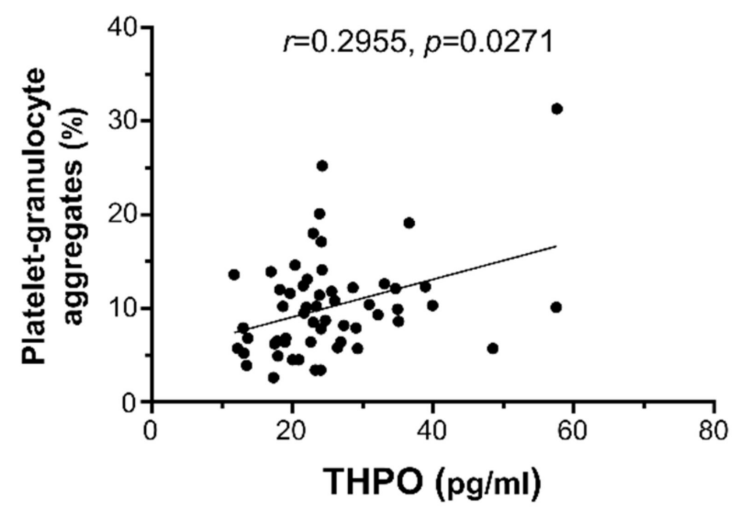

Figure 3. Correlation of THPO plasma levels with ex vivo platelet-monocyte (A) and platelet-granulocyte (B) aggregates. Analyses by Spearman correlation tests; correlation coefficient $r$ - and $p$-values are shown.

Moreover, fasting plasma glucose significantly correlated with ex vivo plateletmonocyte $(r=0.4104, p=0.0017)$ and platelet-granulocyte aggregates $(r=0.2662, p=0.0473)$, but not with platelet P-selectin expression. Finally, we observed a positive correlation between $\mathrm{HbA} 1 \mathrm{c}$ and ex vivo platelet-monocyte aggregation $(r=0.5270, p<0.0001)$, plateletgranulocyte binding $(r=0.3924, p=0.0028)$, and platelet $P$-selectin expression $(r=0.3023$, $p=0.0236)$. 


\subsection{Effect of Plasma of Type 1 Diabetes Mellitus Patients on Platelet Activation In Vitro}

In order to explore the potential involvement of humoral mediators present in the plasma of diabetic patients in enhancing platelet activation, we tested in vitro the effect of plasma from diabetic patients and normal subjects on platelet-leukocyte adhesion in whole blood obtained from healthy donors.

Neither plasma from diabetic patients nor from healthy subjects increased plateletleukocyte binding in whole blood per se, as determined by flow cytometric analysis.

On the contrary, whereas plasma from healthy controls did not increase plateletleukocyte aggregation, plasma from T1DM patients significantly enhanced platelet-monocyte binding induced by EPI (mean \pm SE: $29.32 \% \pm 1.11 \%$ vs. $23.80 \% \pm 0.79 \%$; Figure $4 \mathrm{~A}$ ), but not platelet-granulocyte binding (mean \pm SE: $16.22 \% \pm 2.21 \%$ vs. $14.08 \%$ $\pm 1.84 \%$; Figure $4 \mathrm{~B})$.

A

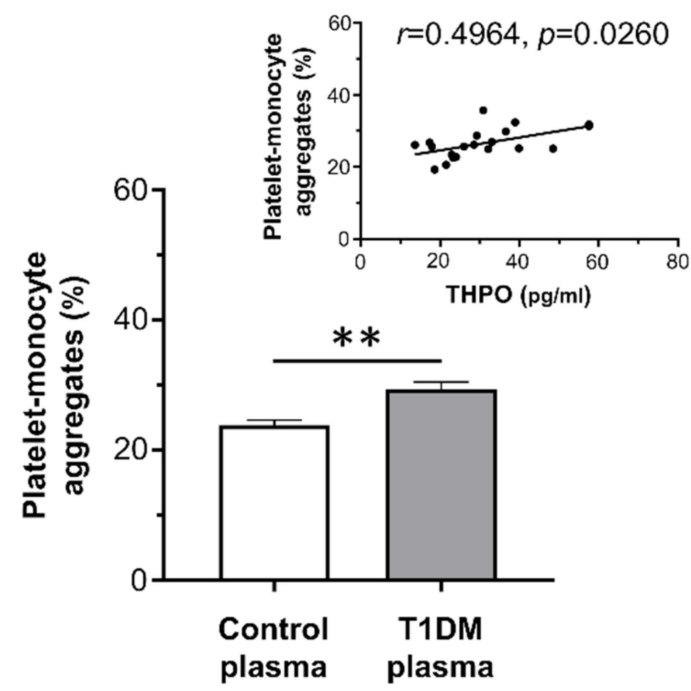

B

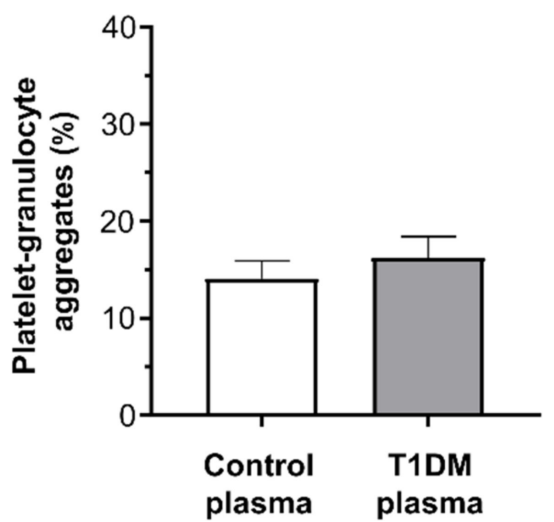

Figure 4. In vitro effect of plasma from healthy subjects and type 1 diabetic patients on epinephrine (EPI)-induced platelet-monocyte (A) and platelet-granulocyte binding (B), as analyzed by flow cytometry in whole blood. Data were represented as mean \pm SE. $p$-values by unpaired $t$-test $\left((\mathbf{A}){ }^{* *} p<0.01 ;(\mathbf{B})\right.$ not statistically significant).

THPO levels measured in vivo significantly correlated with the priming activity exerted in vitro by plasma samples from diabetic patients on platelet-monocyte aggregation $(r=0.4964, p=0.0260$; Figure 4A-insert).

The contribution of THPO to this effect was assessed by inhibiting its biological activity using a recombinant human (rh)THPOR. Pre-treatment of plasma from T1DM patients with the rhTHPOR reduced the priming effect exerted by plasma from type 1 diabetic patients both on platelet-monocyte (mean \pm SE: $22.24 \% \pm 1.71 \%$ vs. $29.32 \% \pm 1.11 \%$; Figure $5 \mathrm{~A}$ ) and platelet-granulocyte binding (mean \pm SE: $14.16 \% \pm 1.85 \%$ vs. $16.22 \% \pm 2.21 \%$; Figure 5B). On the contrary, neither pre-incubation of plasma from healthy subjects with rhTHPOR nor rhTHPOR alone had a significant effect on platelet-leukocyte adhesion (data not shown). 
A

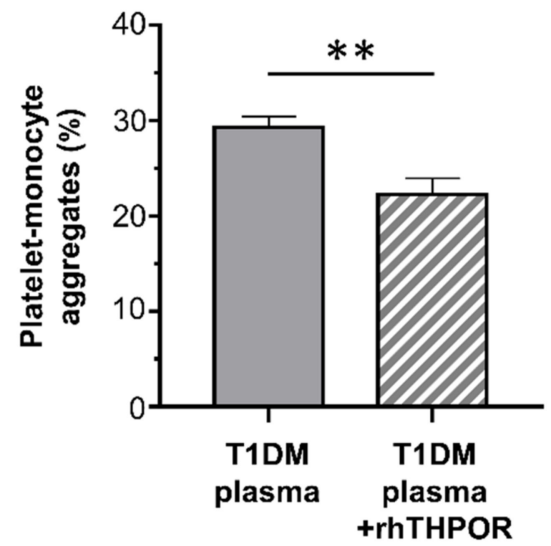

B

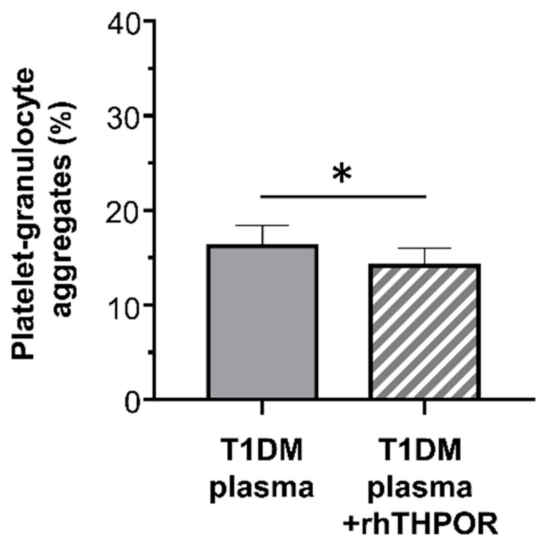

Figure 5. Effect of recombinant human (rh)THPOR on the priming activity induced by plasma from type 1 diabetic patients on EPI-induced platelet-monocyte (A) and platelet-granulocyte binding (B), as analyzed by flow cytometry in whole blood. Data were represented as mean \pm SE. $p$-values by paired $t$-test $\left((\mathbf{A}){ }^{* *} p<0.01 ;(\mathbf{B}) * p<0.05\right)$.

\section{Discussion}

The results of our study suggest that THPO may participate in the pathophysiology of enhanced platelet activation in patients with type 1 diabetes mellitus. We found, indeed, that higher concentrations of circulating THPO were associated with increased indices of platelet activation in vivo in type 1 diabetic patients. Moreover, plasma of type 1 diabetic patients exerted in vitro a priming activity on platelet-leukocyte interaction that appeared to be mediated by plasma THPO contents.

In this study, we have shown that patients with type 1 diabetes mellitus had increased platelet-monocyte and platelet-granulocyte aggregates, together with augmented platelet P-selectin expression, compared to healthy subjects. Our present findings are in line with and confirm previous results demonstrating that type 1 diabetic patients have higher platelet reactivity, which may represent a potential mechanism leading to increased cardiovascular risk [18-22]. In fact, platelet-leukocyte aggregation, which is considered a sensitive measure of platelet activation, also has significant pro-inflammatory and pro-thrombotic consequences, in particular in acute coronary syndrome [23] and sepsis [16,24].

In our study, we also showed that type 1 diabetic patients had a significant, although not dramatic, increase in the circulating levels of THPO. The entity of THPO elevation was modest, as also confirmed by the absence of change in the expression of THPOR on platelet surface. However, since THPO is known to act in cooperation and/or synergy with other mediators, both on platelet activation and in other experimental contests [25], it is reasonable to hypothesize that even slight changes in THPO concentrations may be sufficient to influence these processes.

The description of increased THPO concentrations in type 1 diabetic patients is an innovative result, although the precise origin of the rise in THPO levels in type 1 diabetic patients cannot be definitely clarified.

We think that the observed increase in THPO levels in diabetic patients may probably depend on increased hepatic synthesis sustained by the chronic pro-inflammatory state present in diabetic patients and driven by other inflammatory humoral mediators, for instance, interleukin-6, the main acute-phase reactant produced in the liver [26], which is known to stimulate THPO synthesis in hepatocytes [27].

Much data indicate that THPO levels are primarily regulated by platelet mass $[10,11]$. However, the absence of thrombocytopenia in our patient population and the lack of correlation with MPV and PDW, which are considered markers of increased platelet turn-over, seem to exclude this causative mechanism in patients with type 1 diabetes mellitus. Still, 
previous studies, in populations larger than ours, have shown that diabetic patients have increased indices of platelet turn-over [28-30], and the consequent increased production of larger and more reactive new platelets has been related to increased platelet reactivity [31].

Finally, since we found a positive correlation between THPO and both plateletleukocyte binding and platelet P-selectin expression in vivo, it can also be hypothesized that the augmented THPO concentrations are, at least partially, due to increased release by platelets themselves. Activated platelets, indeed, are known to release full-length biological active THPO upon stimulation [32]. Of note, THPO released by activated platelets may also concur to further increase inflammation by activating other cell types, for instance, stimulating motility and angiogenesis in endothelial cells [33] and enhancing interleukin-8 and reactive oxygen species release from neutrophils [34].

Several studies have shown that platelet activation is augmented in diabetic patients [35-40]. The pathophysiologic mechanisms involved in this phenomenon, although not fully elucidated, include, for instance, (a) the reduced production and activity of nitric oxide induced by oxidative stress [6,41,42]; (b) the generalized status of systemic hyperinflammation, sustained by different mediators, present in diabetic patients $[43,44]$; and (c) qualitative and quantitative changes in coagulation factors [45,46], which contribute to the pro-thrombotic environment through the formation of compact fibrin networks and depressed fibrinolytic activity $[47,48]$.

A main factor contributing to the enhanced platelet reactivity is represented by metabolic factors. Both acute and chronic changes in plasma glucose correlate, indeed, with increased expression of platelet activation markers, such as P-selectin and CD40-ligand, on platelet surface [6,49-53]. Hyperglycaemia may contribute to increase platelet activation via different mechanisms, which include augmented glycation of platelet surface proteins, which decreases membrane fluidity and increases platelet adhesion [54,55], and the direct osmotic effect of glucose [56], which can activate protein kinase C [57] and the nitric oxide/cyclic nucleotide pathway [58,59]. Further confirming these pieces of evidence, early intensive glucose control decreases platelet reactivity in patients with acute coronary syndrome and hyperglycaemia [60], and it reduces mortality in diabetic patients with acute coronary syndrome at 3.4 years of follow-up [61].

In our study, we found a significant correlation between THPO concentrations and both fasting glucose and $\mathrm{HbA1c}$ levels. These data confirm the causal relationship between metabolic control and platelet activation in type 1 diabetic patients and indicate THPO as a new mediator involved in the pathogenesis of this phenomenon, with potential therapeutic implications.

In order to study the contribution of THPO to increased platelet activation in type 1 diabetic patients, we evaluated the effects of adding plasma samples from diabetic patients to platelets of healthy subjects in vitro and inhibiting THPO biological activity by using rhTHPOR. In these experimental conditions, plasma from type 1 diabetic patients, but not from healthy subjects, enhanced platelet-monocyte and platelet-granulocyte binding in blood samples from healthy donors. The contribution of THPO to this priming effect is suggested by (1) the correlation analysis showing that THPO levels and leucocyte-platelet binding induced by patient plasma samples in whole blood consensually increased, and (2) the inhibitory effect of the rhTHPOR.

Taken together, our in vivo and in vitro results support the hypothesis that the elevated concentrations of THPO present in the circulation of diabetic patients may facilitate platelet activation by sensitizing platelets to the action of other agonists, thus augmenting the cardiovascular risk of these patients. This model is consistent with what we had previously observed in other clinical conditions characterized by increased thrombotic risk (acute coronary syndromes, sepsis, burn injury, cigarette smoking), as well as with the priming effect induced in vivo by THPO infusion in non-human primates [62].

Finally, we can speculate that this effect may be implicated in the pathogenesis of the "reduced sensitivity" of platelets from diabetic patients to oral antiplatelet agents used for primary and secondary prevention, a phenomenon that is particularly prevalent in diabetic 
patients [63-65] and could explain the reduced efficacy of aspirin treatment in this patient population [63,66-68].

In conclusion, our results suggest that increased THPO levels may enhance platelet activation in patients with type 1 diabetes mellitus and contribute to increased cardiovascular risk in this patient population.

\section{Materials and Methods}

\subsection{Patients and Healthy Controls}

This was a case-control study performed on 28 diabetic patients attending the Department of Endocrinology, Diabetology and Metabolism-Diabetes Center at A.O.U. Città della Salute e della Scienza di Torino, Italy. Twenty-eight healthy subjects matched in a 1:1 ratio for age and gender to cases were also enrolled. None of both diabetic patients and healthy subjects had acute or chronic infections, preproliferative or proliferative retinopathy, diabetic neuropathy, cardiovascular disease, hypertension, or drug treatment (except insulin in diabetic patients) at the time of enrolment.

Exclusion criteria were as follows: age $<18$ years or $>50$ years; current smoker; treatment with regular medication (other than insulin for T1DM group) or anti-platelet agent assumption within the preceding 2 weeks; clinical evidence of any condition associated with increased levels of acute phase proteins (infectious diseases, surgery within the previous 1 month); known hematological diseases affecting coagulation, platelet, or THPO production; malignancies; body mass index $>30 \mathrm{~kg} / \mathrm{m}^{2}$; hypercholesterolemia; hypertension; cardiovascular diseases; micro/macroalbuminuria; neuropathy; proliferative retinopathy; and renal or hepatic insufficiency.

The study was approved by the Institutional Ethics Committee of A.O.U. Città della Salute e della Scienza di Torino (\#0076185). Written informed consent was obtained from all patients; all samples were anonymously coded in accordance with the Declaration of Helsinki.

\subsection{Blood Collection Protocol}

Venipuncture was performed with a 19-gauge butterfly infusion set, without venous stasis. After discarding the first $4 \mathrm{~mL}$, blood was drawn into Vacutainers containing EDTA or $3.8 \%$ trisodium citrate, as appropriate.

To obtain plasma samples, EDTA-anticoagulated tubes were first centrifuged at $1600 \times g$ for $10 \mathrm{~min}$ at $4{ }^{\circ} \mathrm{C}$, then at $12,500 \times g$ for $10 \mathrm{~min}$ at $4{ }^{\circ} \mathrm{C}$, filtered through $0.22 \mu \mathrm{m}$ pores, and immediately frozen and stored at $-70^{\circ} \mathrm{C}$ until analysis.

Platelet-rich plasma (PRP) was prepared by centrifugation of $3.8 \%$ trisodium citrateanticoagulated blood for $15 \mathrm{~min}$ at $180 \times g$ [12].

\subsection{Leucocyte-Platelet Adhesion and Platelet THPOR Expression Ex Vivo}

Platelet-leukocyte aggregates were analyzed by three-color staining of whole blood samples, as previously described [15]. Briefly, blood was diluted 1:1 with Tyrodes's HEPESbuffered saline ( $\mathrm{pH}$ 7.4); added to a mixture of FITC-conjugated anti-CD45 (Beckman Coulter, Miami, FL, USA), ECD-conjugated anti-CD14 (Beckman Coulter), and PE-conjugated anti-CD41 (Dako Cytomation, Glostrup, Denmark) monoclonal antibodies; and incubated $15 \mathrm{~min}$ at room temperature. Cells were then fixed with $1 \%$ paraformaldehyde and resuspended in $0.5 \mathrm{~mL}$ of PBS, after removal of erythrocytes by hypotonic lysis. Samples were analyzed on the EPICS-XL flow cytometer (Coulter Corp, Hialeah, FL, USA) using adequate compensation for different fluorochromes. Total leukocytes were identified by their positive staining with anti-CD45, and lymphocyte, granulocyte, and monocyte populations were discriminated on the ground of CD45 versus side scatter. The percentage of leukocyte sub-groups co-expressing CD45-CD41 (granulocytes-platelets) or CD14-CD41 (monocytes-platelets) over the total population of leukocytes expressing CD45 or CD14 was used as an index of platelet-leukocyte adhesion [15]. P-selectin expression was evaluated in whole blood using a mixture of FITC-conjugated anti-CD62P/P-selectin (Ancell Corpora- 
tion, Bayport, MN, USA) or the appropriate isotypic control, and PE-conjugated anti-CD41 monoclonal antibodies. Platelets were identified by their characteristic light scatter and the positive signal provided by the platelet marker PE-anti-CD41 monoclonal antibody.

Platelet THPOR surface expression was examined in fixed PRP using an anti-THPOR monoclonal antibody (R\&D Systems Inc., Minneapolis, MN, USA), followed by Alexa Fluor 488-conjugated anti-mouse IgG monoclonal antibody (Molecular Probes, Eugene, OR, USA).

\subsection{Leucocyte-Platelet Adhesion In Vitro}

In order to explore the potential involvement of humoral mediators present in the plasma of diabetic patients in enhancing platelet activation, we tested in vitro the effect of plasma from diabetic patients and normal subjects on platelet-leukocyte adhesion in whole blood obtained from healthy donors.

For in vitro experiments, $100 \mu \mathrm{L}$ of diluted blood from healthy adult donors was pre-incubated at $37^{\circ} \mathrm{C}$ with $25 \mu \mathrm{L}$ of plasma of healthy or T1DM subjects for $5 \mathrm{~min}$ and then stimulated with EPI ( $4 \mu \mathrm{mol} / \mathrm{L}$; Helena Laboratories, Beaumont, TX, USA). Samples were then processed and analyzed as described above. In separate experiments, the plasma was incubated with rhTHPOR $\left(2.5 \mu \mathrm{g} / \mathrm{mL}\right.$; R\&D Systems Inc.) for $5 \mathrm{~min}$ at $37^{\circ} \mathrm{C}$; the mixture of sample and rhTHPOR was added to whole blood, further incubated for $5 \mathrm{~min}$ at $37^{\circ} \mathrm{C}$ and stimulated with EPI.

\subsection{Biochemical Analyses}

Plasma glucose was assayed by the glucose oxidase method (Beckman II, Glucose Analyser, Fullerton $\mathrm{CA}$ ); HbAlc was determined by high-performance liquid chromatography (DIAMAT, Bio-Rad, Richmond, CA, USA); plasma triglycerides and total cholesterol were determined enzymatically (Boehringer Mannheim, Germany). High-density lipoprotein (HDL)-cholesterol was measured by precipitation with heparin and $\mathrm{MgCl}_{2}$ on whole plasma, whereas low-density lipoprotein (LDL)-cholesterol was calculated by Friedewald's formula.

THPO levels in plasma were quantified using a specific ELISA kit (R\&D Systems) following the manufacturer's instructions. The lower detection limit of the assay was $7.45 \mathrm{pg} / \mathrm{mL}$.

\subsection{Statistical Analysis}

Statistically significant differences between datasets were evaluated by the MannWhitney rank sum test or unpaired $t$-test, and paired $t$-tests, as appropriate. The Spearman correlation test was used to investigate the relationships between variables.

$p<0.05$ was considered statistically significant.

Statistical analysis was performed with the GraphPad Prism 7 package (GraphPad Software, La Jolla, CA, USA).

Author Contributions: Conceptualization, P.C.-P., G.G., I.R., G.M. and E.L.; methodology, O.B., B.V., M.S. and B.L.; formal analysis, B.V. and O.B.; investigation, O.B., B.V., M.S. and B.L.; resources, G.G.; data curation, B.V. and O.B.; writing—original draft preparation, O.B., B.V. and E.L.; writing-review and editing, P.C.-P., G.G., I.R., G.M. and E.L.; visualization, B.V. and O.B.; supervision, E.L.; project administration, G.M. and E.L.; funding acquisition, G.M. and E.L. All authors have read and agreed to the published version of the manuscript.

Funding: This research was funded by "Research Fund ex-60\%", University of Turin, to E.L. and G.M.

Institutional Review Board Statement: The study was conducted according to the guidelines of the Declaration of Helsinki and approved by the Institutional Ethics Committee of A.O.U. Città della Salute e della Scienza di Torino (protocol no. 0076185; approved on the 19 October 2007).

Informed Consent Statement: Informed consent was obtained from all subjects involved in the study. 
Data Availability Statement: The data presented in this study are available on reasonable request from the corresponding author. The data are not publicly available due to privacy concerns.

Conflicts of Interest: The authors declare no conflict of interests.

\section{References}

1. Shi, M.; Tang, R.; Huang, F.; Zhong, T.; Chen, Y.; Li, X.; Zhou, Z. Cardiovascular disease in patients with type 1 diabetes: Early evaluation, risk factors and possible relation with cardiac autoimmunity. Diabetes Metab. Res. Rev. 2020. [CrossRef]

2. Vergès, B. Cardiovascular disease in type 1 diabetes: A review of epidemiological data and underlying mechanisms. Diabetes Metab. 2020, 46, 442-449. [CrossRef] [PubMed]

3. Bratseth, V.; Margeirsdottir, H.D.; Chiva-Blanch, G.; Heier, M.; Solheim, S.; Arnesen, H.; Dahl-Jørgensen, K.; Seljeflot, I. Annexin V+ Microvesicles in Children and Adolescents with Type 1 Diabetes: A Prospective Cohort Study. J. Diabetes Res. 2020, 2020, 7216863. [CrossRef] [PubMed]

4. Orchard, T.J.; Costacou, T. Cardiovascular complications of type 1 diabetes: Update on the renal link. Acta Diabetol. 2017, 54, 325-334. [CrossRef] [PubMed]

5. Shah, V.N.; Bailey, R.; Wu, M.; Foster, N.C.; Pop-Busui, R.; Katz, M.; Crandall, J.; Bacha, F.; Nadeau, K.; Libman, I.; et al. Risk Factors for Cardiovascular Disease (CVD) in Adults with Type 1 Diabetes: Findings from Prospective Real-life T1D Exchange Registry. J. Clin. Endocrinol. Metab. 2020, 105. [CrossRef]

6. Schneider, D.J. Factors contributing to increased platelet reactivity in people with diabetes. Diabetes Care 2009, 32, 525-527. [CrossRef] [PubMed]

7. Zahran, A.M.; El-Badawy, O.; Mohamad, I.L.; Tamer, D.M.; Abdel-Aziz, S.M.; Elsayh, K.I. Platelet Activation and PlateletLeukocyte Aggregates in Type I Diabetes Mellitus. Clin. Appl. Thromb. Hemost. 2018, 24, 230S-239S. [CrossRef]

8. Angiolillo, D.J. Antiplatelet therapy in diabetes: Efficacy and limitations of current treatment strategies and future directions. Diabetes Care 2009, 32, 531-540. [CrossRef]

9. Rivas Rios, J.R.; Franchi, F.; Rollini, F.; Angiolillo, D.J. Diabetes and antiplatelet therapy: From bench to bedside. Cardiovasc. Diagn. Ther. 2018, 8, 594-609. [CrossRef]

10. Kaushansky, K. Thrombopoietin: A tool for understanding thrombopoiesis. J. Thromb. Haemost. 2003, 1, 1587-1592. [CrossRef]

11. Kuter, D.J.; Begley, C.G. Recombinant human thrombopoietin: Basic biology and evaluation of clinical studies. Blood 2002, 100, 3457-3469. [CrossRef] [PubMed]

12. Montrucchio, G.; Brizzi, M.F.; Calosso, G.; Marengo, S.; Pegoraro, L.; Camussi, G. Effects of recombinant human megakaryocyte growth and development factor on platelet activation. Blood 1996, 87, 2762-2768. [CrossRef] [PubMed]

13. Oda, A.; Miyakawa, Y.; Druker, B.J.; Ozaki, K.; Yabusaki, K.; Shirasawa, Y.; Handa, M.; Kato, T.; Miyazaki, H.; Shimosaka, A.; et al. Thrombopoietin primes human platelet aggregation induced by shear stress and by multiple agonists. Blood 1996, 87, 4664-4670. [CrossRef]

14. Tibbles, H.E.; Navara, C.S.; Hupke, M.A.; Vassilev, A.O.; Uckun, F.M. Thrombopoietin induces p-selectin expression on platelets and subsequent platelet/leukocyte interactions. Biochem. Biophys. Res. Commun. 2002, 292, 987-991. [CrossRef]

15. Lupia, E.; Bosco, O.; Bergerone, S.; Dondi, A.E.; Goffi, A.; Oliaro, E.; Cordero, M.; Del Sorbo, L.; Trevi, G.; Montrucchio, G. Thrombopoietin Contributes to Enhanced Platelet Activation in Patients With Unstable Angina. J. Am. Coll. Cardiol. 2006, 48, 2195-2203. [CrossRef]

16. Lupia, E.; Bosco, O.; Mariano, F.; Dondi, A.E.; Goffi, A.; Spatola, T.; Cuccurullo, A.; Tizzani, P.; Brondino, G.; Stella, M.; et al. Elevated thrombopoietin in plasma of burned patients without and with sepsis enhances platelet activation. J. Thromb. Haemost. 2009, 7, 1000-1008. [CrossRef]

17. Lupia, E.; Bosco, O.; Goffi, A.; Poletto, C.; Locatelli, S.; Spatola, T.; Cuccurullo, A.; Montrucchio, G. Thrombopoietin contributes to enhanced platelet activation in cigarette smokers. Atherosclerosis 2010, 210, 314-319. [CrossRef] [PubMed]

18. Keaney, J.F.; Loscalzo, J. Diabetes, oxidative stress, and platelet activation. Circulation 1999, 99, 189-191. [CrossRef]

19. Hu, H.; Li, N.; Yngen, M.; Ostenson, C.G.; Wallén, N.H.; Hjemdahl, P. Enhanced leukocyte-platelet cross-talk in Type 1 diabetes mellitus: Relationship to microangiopathy. J. Thromb. Haemost. 2004, 2, 58-64. [CrossRef]

20. Véricel, E.; Januel, C.; Carreras, M.; Moulin, P.; Lagarde, M. Diabetic patients without vascular complications display enhanced basal platelet activation and decreased antioxidant status. Diabetes 2004, 53, 1046-1051. [CrossRef]

21. Wisinski, J.A.; Kimple, M.E. Platelet Dysfunction in Type 1 Diabetes: Stressing the Thromboxanes. Diabetes 2016, 65, 349-351. [CrossRef]

22. Harding, S.A.; Sommerfield, A.J.; Sarma, J.; Twomey, P.J.; Newby, D.E.; Frier, B.M.; Fox, K.A. Increased CD40 ligand and platelet-monocyte aggregates in patients with type 1 diabetes mellitus. Atherosclerosis 2004, 176, 321-325. [CrossRef]

23. Freedman, J.E.; Loscalzo, J. Platelet-monocyte aggregates: Bridging thrombosis and inflammation. Circulation 2002, 105, $2130-2132$. [CrossRef]

24. Lupia, E.; Goffi, A.; Bosco, O.; Montrucchio, G. Thrombopoietin as biomarker and mediator of cardiovascular damage in critical diseases. Mediators Inflamm. 2012, 2012, 390892. [CrossRef] [PubMed]

25. Lupia, E.; Spatola, T.; Cuccurullo, A.; Bosco, O.; Mariano, F.; Pucci, A.; Ramella, R.; Alloatti, G.; Montrucchio, G. Thrombopoietin modulates cardiac contractility in vitro and contributes to myocardial depressing activity of septic shock serum. Basic Res. Cardiol. 2010, 105, 609-620. [CrossRef] [PubMed] 
26. Wolber, E.M.; Jelkmann, W. Interleukin-6 increases thrombopoietin production in human hepatoma cells HepG2 and Hep3B. J. Interferon Cytokine Res. 2000, 20, 499-506. [CrossRef]

27. Wolber, E.M.; Haase, B.; Jelkmann, W. Thrombopoietin production in human hepatic cell cultures (HepG2) is resistant to IFN-alpha, IFN-beta, and IFN-gamma treatment. J. Interferon Cytokine Res. 2002, 22, 1185-1189. [CrossRef]

28. Coban, E.; Bostan, F.; Ozdogan, M. The mean platelet volume in subjects with impaired fasting glucose. Platelets 2006, 17, 67-69. [CrossRef]

29. Santilli, F.; Formoso, G.; Sbraccia, P.; Averna, M.; Miccoli, R.; Di Fulvio, P.; Ganci, A.; Pulizzi, N.; Lattanzio, S.; Ciabattoni, G.; et al. Postprandial hyperglycemia is a determinant of platelet activation in early type 2 diabetes mellitus. J. Thromb. Haemost. 2010, 8 , 828-837. [CrossRef]

30. Guthikonda, S.; Lev, E.I.; Patel, R.; DeLao, T.; Bergeron, A.L.; Dong, J.F.; Kleiman, N.S. Reticulated platelets and uninhibited COX-1 and COX-2 decrease the antiplatelet effects of aspirin. J. Thromb. Haemost. 2007, 5, 490-496. [CrossRef] [PubMed]

31. Guthikonda, S.; Alviar, C.L.; Vaduganathan, M.; Arikan, M.; Tellez, A.; DeLao, T.; Granada, J.F.; Dong, J.F.; Kleiman, N.S.; Lev, E.I. Role of reticulated platelets and platelet size heterogeneity on platelet activity after dual antiplatelet therapy with aspirin and clopidogrel in patients with stable coronary artery disease. J. Am. Coll. Cardiol. 2008, 52, 743-749. [CrossRef]

32. Folman, C.C.; Linthorst, G.E.; van Mourik, J.; van Willigen, G.; de Jonge, E.; Levi, M.; de Haas, M.; von dem Borne, A.E. Platelets release thrombopoietin (Tpo) upon activation: Another regulatory loop in thrombocytopoiesis? Thromb. Haemost. 2000, 83, 923-930. [CrossRef] [PubMed]

33. Brizzi, M.F.; Battaglia, E.; Montrucchio, G.; Dentelli, P.; Del Sorbo, L.; Garbarino, G.; Pegoraro, L.; Camussi, G. Thrombopoietin stimulates endothelial cell motility and neoangiogenesis by a platelet-activating factor-dependent mechanism. Circ. Res. 1999, 84, 785-796. [CrossRef]

34. Brizzi, M.F.; Battaglia, E.; Rosso, A.; Strippoli, P.; Montrucchio, G.; Camussi, G.; Pegoraro, L. Regulation of polymorphonuclear cell activation by thrombopoietin. J. Clin. Investig. 1997, 99, 1576-1584. [CrossRef] [PubMed]

35. Ferroni, P.; Basili, S.; Falco, A.; Davì, G. Platelet activation in type 2 diabetes mellitus. J. Thromb. Haemost. 2004, 2, $1282-1291$. [CrossRef]

36. Rollini, F.; Franchi, F.; Muñiz-Lozano, A.; Angiolillo, D.J. Platelet function profiles in patients with diabetes mellitus. J. Cardiovasc. Transl. Res. 2013, 6, 329-345. [CrossRef] [PubMed]

37. Santilli, F.; Simeone, P.; Liani, R.; Davì, G. Platelets and diabetes mellitus. Prostaglandins Other Lipid Mediat. 2015, 120, 28-39. [CrossRef]

38. Russo, I.; Penna, C.; Musso, T.; Popara, J.; Alloatti, G.; Cavalot, F.; Pagliaro, P. Platelets, diabetes and myocardial ischemia/reperfusion injury. Cardiovasc. Diabetol. 2017, 16, 71. [CrossRef]

39. Carrizzo, A.; Izzo, C.; Oliveti, M.; Alfano, A.; Virtuoso, N.; Capunzo, M.; Di Pietro, P.; Calabrese, M.; de Simone, E.; Sciarretta, S.; et al. The Main Determinants of Diabetes Mellitus Vascular Complications: Endothelial Dysfunction and Platelet Hyperaggregation. Int. J. Mol. Sci. 2018, 19, 2968. [CrossRef]

40. Barale, C.; Cavalot, F.; Frascaroli, C.; Bonomo, K.; Morotti, A.; Guerrasio, A.; Russo, I. Association between High On-Aspirin Platelet Reactivity and Reduced Superoxide Dismutase Activity in Patients Affected by Type 2 Diabetes Mellitus or Primary Hypercholesterolemia. Int. J. Mol. Sci. 2020, 21, 4983. [CrossRef]

41. Anfossi, G.; Mularoni, E.M.; Burzacca, S.; Ponziani, M.C.; Massucco, P.; Mattiello, L.; Cavalot, F.; Trovati, M. Platelet resistance to nitrates in obesity and obese NIDDM, and normal platelet sensitivity to both insulin and nitrates in lean NIDDM. Diabetes Care 1998, 21, 121-126. [CrossRef] [PubMed]

42. Santilli, F.; Lapenna, D.; La Barba, S.; Davì, G. Oxidative stress-related mechanisms affecting response to aspirin in diabetes mellitus. Free Radic Biol. Med. 2015, 80, 101-110. [CrossRef] [PubMed]

43. Calverley, D.C.; Hacker, M.R.; Loda, K.A.; Brass, E.; Buchanan, T.A.; Tsao-Wei, D.D.; Groshen, S. Increased platelet Fc receptor expression as a potential contributing cause of platelet hypersensitivity to collagen in diabetes mellitus. Br. J. Haematol. 2003, 121, 139-142. [CrossRef] [PubMed]

44. Lim, H.S.; Blann, A.D.; Lip, G.Y. Soluble CD40 ligand, soluble P-selectin, interleukin-6, and tissue factor in diabetes mellitus: Relationships to cardiovascular disease and risk factor intervention. Circulation 2004, 109, 2524-2528. [CrossRef] [PubMed]

45. Alzahrani, S.H.; Ajjan, R.A. Coagulation and fibrinolysis in diabetes. Diab. Vasc. Dis. Res. 2010, 7, 260-273. [CrossRef]

46. Kearney, K.; Tomlinson, D.; Smith, K.; Ajjan, R. Hypofibrinolysis in diabetes: A therapeutic target for the reduction of cardiovascular risk. Cardiovasc. Diabetol. 2017, 16, 34. [CrossRef]

47. Ajjan, R.A.; Gamlen, T.; Standeven, K.F.; Mughal, S.; Hess, K.; Smith, K.A.; Dunn, E.J.; Anwar, M.M.; Rabbani, N.; Thornalley, P.J.; et al. Diabetes is associated with posttranslational modifications in plasminogen resulting in reduced plasmin generation and enzyme-specific activity. Blood 2013, 122, 134-142. [CrossRef]

48. Sumaya, W.; Wallentin, L.; James, S.K.; Siegbahn, A.; Gabrysch, K.; Bertilsson, M.; Himmelmann, A.; Ajjan, R.A.; Storey, R.F. Fibrin clot properties independently predict adverse clinical outcome following acute coronary syndrome: A PLATO substudy. Eur. Heart J. 2018, 39, 1078-1085. [CrossRef]

49. Yngen, M.; Ostenson, C.G.; Li, N.; Hjemdahl, P.; Wallén, N.H. Acute hyperglycemia increases soluble P-selectin in male patients with mild diabetes mellitus. Blood Coagul. Fibrinolysis 2001, 12, 109-116. [CrossRef]

50. Vaidyula, V.R.; Rao, A.K.; Mozzoli, M.; Homko, C.; Cheung, P.; Boden, G. Effects of hyperglycemia and hyperinsulinemia on circulating tissue factor procoagulant activity and platelet CD40 ligand. Diabetes 2006, 55, 202-208. [CrossRef] 
51. Vaidyula, V.R.; Boden, G.; Rao, A.K. Platelet and monocyte activation by hyperglycemia and hyperinsulinemia in healthy subjects. Platelets 2006, 17, 577-585. [CrossRef]

52. Yngen, M.; Norhammar, A.; Hjemdahl, P.; Wallén, N.H. Effects of improved metabolic control on platelet reactivity in patients with type 2 diabetes mellitus following coronary angioplasty. Diab. Vasc. Dis. Res. 2006, 3, 52-56. [CrossRef]

53. Undas, A.; Wiek, I.; Stêpien, E.; Zmudka, K.; Tracz, W. Hyperglycemia is associated with enhanced thrombin formation, platelet activation, and fibrin clot resistance to lysis in patients with acute coronary syndrome. Diabetes Care 2008, 31, 1590-1595. [CrossRef]

54. Winocour, P.D.; Watala, C.; Perry, D.W.; Kinlough-Rathbone, R.L. Decreased platelet membrane fluidity due to glycation or acetylation of membrane proteins. Thromb. Haemost. 1992, 68, 577-582. [CrossRef] [PubMed]

55. Watala, C.; Golański, J.; Boncler, M.A.; Pietrucha, T.; Gwoździński, K. Membrane lipid fluidity of blood platelets: A common denominator that underlies the opposing actions of various agents that affect platelet activation in whole blood. Platelets 1998, 9 , 315-327. [CrossRef] [PubMed]

56. Keating, F.K.; Sobel, B.E.; Schneider, D.J. Effects of increased concentrations of glucose on platelet reactivity in healthy subjects and in patients with and without diabetes mellitus. Am. J. Cardiol 2003, 92, 1362-1365. [CrossRef] [PubMed]

57. Assert, R.; Scherk, G.; Bumbure, A.; Pirags, V.; Schatz, H.; Pfeiffer, A.F. Regulation of protein kinase C by short term hyperglycaemia in human platelets in vivo and in vitro. Diabetologia 2001, 44, 188-195. [CrossRef] [PubMed]

58. Massucco, P.; Mattiello, L.; Russo, I.; Traversa, M.; Doronzo, G.; Anfossi, G.; Trovati, M. High glucose rapidly activates the nitric oxide/cyclic nucleotide pathway in human platelets via an osmotic mechanism. Thromb. Haemost. 2005, 93, 517-526. [CrossRef]

59. Bergandi, L.; Cordero, M.; Anselmino, M.; Ferraro, G.; Ravera, L.; Dalmasso, P.; Moiraghi, C.; Trevi, G.P.; Ghigo, D.; Bosia, A.; et al. Altered nitric oxide/cGMP platelet signaling pathway in platelets from patients with acute coronary syndromes. Clin. Res. Cardiol. 2010, 99, 557-564. [CrossRef]

60. Vivas, D.; García-Rubira, J.C.; Bernardo, E.; Angiolillo, D.J.; Martín, P.; Calle-Pascual, A.; Núñez-Gil, I.; Macaya, C.; FernándezOrtiz, A. Effects of intensive glucose control on platelet reactivity in patients with acute coronary syndromes. Results of the CHIPS Study ("Control de Hiperglucemia y Actividad Plaquetaria en Pacientes con Sindrome Coronario Agudo"). Heart 2011, 97, 803-809. [CrossRef] [PubMed]

61. Malmberg, K. Prospective randomised study of intensive insulin treatment on long term survival after acute myocardial infarction in patients with diabetes mellitus. DIGAMI (Diabetes Mellitus, Insulin Glucose Infusion in Acute Myocardial Infarction) Study Group. BMJ 1997, 314, 1512-1515. [CrossRef]

62. Harker, L.A.; Marzec, U.M.; Hunt, P.; Kelly, A.B.; Tomer, A.; Cheung, E.; Hanson, S.R.; Stead, R.B. Dose-response effects of pegylated human megakaryocyte growth and development factor on platelet production and function in nonhuman primates. Blood 1996, 88, 511-521. [CrossRef]

63. Zaccardi, F.; Rizzi, A.; Petrucci, G.; Ciaffardini, F.; Tanese, L.; Pagliaccia, F.; Cavalca, V.; Ciminello, A.; Habib, A.; Squellerio, I.; et al. In Vivo Platelet Activation and Aspirin Responsiveness in Type 1 Diabetes. Diabetes 2016, 65, 503-509. [CrossRef]

64. Colwell, J.A. Is aspirin effective in diabetic patients? Yes. J. Thromb. Haemost. 2005, 3, 2612-2614. [CrossRef] [PubMed]

65. Nicolucci, A.; de Berardis, G.; Sacco, M.; Tognoni, G. AHA/ADA vs. ESC/EASD recommendations on aspirin as a primary prevention strategy in people with diabetes: How the same data generate divergent conclusions. Eur. Heart J. 2007, 28, 1925-1927. [CrossRef]

66. Mehta, S.S.; Silver, R.J.; Aaronson, A.; Abrahamson, M.; Goldfine, A.B. Comparison of aspirin resistance in type 1 versus type 2 diabetes mellitus. Am. J. Cardiol. 2006, 97, 567-570. [CrossRef]

67. Fitzgerald, R.; Pirmohamed, M. Aspirin resistance: Effect of clinical, biochemical and genetic factors. Pharmacol. Ther. 2011, 130, 213-225. [CrossRef] [PubMed]

68. Simpson, S.H.; Abdelmoneim, A.S.; Omran, D.; Featherstone, T.R. Prevalence of high on-treatment platelet reactivity in diabetic patients treated with aspirin. Am. J. Med. 2014, 127, 95.e1-95.e9. [CrossRef] [PubMed] 